\title{
From Insight to Modulation of CXCR4 and ACKR3 (CXCR7) Function
}

\author{
Martine J. Smit and Jacqueline E. van Muijlwijk-Koezen \\ Division of Medicinal Chemistry, Amsterdam Institute for Molecules Medicines and Systems, Vrije Universiteit Amsterdam, \\ Amsterdam, The Netherlands
}

Received September 25, 2019; accepted September 27, 2019

\section{ABSTRACT}

Chemokine receptors CXCR4 and atypical chemokine receptor 3 (ACKR3/CXCR7) are highly expressed in a range of tumors. Yet, their role in cancer progression is not well understood. This minireview series encompasses seven comprehensive reviews focusing on modulators (small molecules, pepducins, antibodies), structural aspects, spatio-temporal signaling properties, and phosphorylation/interactome of CXCR4 and ACKR3. Moreover, different (patho)physiologic aspects and roles of these receptors in immunologic and oncogenic processes are discussed.

\section{SIGNIFICANCE STATEMENT}

CXCR4 and atypical chemokine receptor 3 are two oncogenic $\mathrm{G}$ protein-coupled receptors that are highly upregulated in various tumors. Insight into the signalling properties of these receptors and the availability of modulators targeting these receptors are essential to assess their role in cancer.
There is increasing evidence that $\mathrm{G}$ protein-coupled receptors (GPCRs) and their associated signaling cascades are involved in both cancer progression and metastasis (O'Hayre et al., 2013). As oncogenic GPCRs are likely to be amenable to manipulation via drugs, they are ideal therapeutic targets. In the ITN Marie Curie Training network Oncogenic GPCR Network of Excellence and Training (ONCORNET), we have been studying two oncogenic GPCRs: the chemokine receptors CXCR4 and atypical chemokine receptor 3 (ACKR3), formerly referred to as CXCR7. These receptors are highly expressed in a range of tumors, yet their role in cancer progression is not well understood (Peled et al., 2012; Sánchez-Martín et al., 2013). Early-stage researchers and principal investigators of the ONCORNET consortium, from different academic and industrial partners throughout Europe, joined forces investigating these receptors and have contributed to this minireview series. This mini series encompasses seven comprehensive reviews focusing on modulators (small molecules, pepducins, antibodies), structural aspects, spatio-temporal signaling properties, and phosphorylation/interactome of CXCR4 and ACKR3. Moreover, different (patho)physiologic aspects and roles of these receptors in oncogenic and immunologic processes are discussed.

https://doi.org/10.1124/mol.119.118364.
Small-molecule and known modulators of CXCR4 and ACKR3 are discussed in detail based on common structural motifs and available crystal structures (Adlere et al., 2019). Selectivity, receptor activation, and signaling output of small molecules, chemokines, pepducins, and peptide ligands are reviewed. In addition, the development of radiolabeled and fluorescently labeled tool compounds, enabling visualization of ligand binding and receptor characterization in vitro and in vivo, is addressed. These receptors are also attractive targets for monoclonal antibodies, antibody fragments, and variants directed at CXCR4 and ACKR3. The advantageous functional properties, versatile applications of these antibodies, and derivatives as research tools and use in the clinic are outlined and discussed in another review (Bobkov et al., 2019). In the last decade, significant progress has been made in obtaining crystal structures of GPCRs. One of the reviews summarizes the available data on chemokine receptor crystal structures and discusses the numerous applications, challenges, and pitfalls from chemokine receptor structures to support studies of molecular pharmacologists (Arimont et al., 2019).

Another review focuses on the context-dependent signaling of CXCR4 and ACKR3, an atypical chemokine receptor, generally reported not to activate $G$ proteins (Heuninck et al., 2019). Yet, both receptors can also form heteromers with other GPCRs, not only chemokine receptors, and other proteins, which results in differential signaling compared with

ABBREVIATIONS: ACKR3, atypical chemokine receptor 3; GPCR, G protein-coupled receptor; ONCORNET, Oncogenic GPCR Network of Excellence and Training. 
that of the monomeric subunits. Moreover, their location during cancer progression may change, acquiring different signaling properties. Next, one review describes state-of-theart methods for the identification of GPCR-interacting proteins and GPCR-phosphorylated sites (Fumagalli et al., 2019). As for CXCR4 and ACKR3, post-translational modifications and previously identified CXCR4- or ACKR3-interacting proteins are outlined. Studies highlighting the importance of the reciprocal influence of CXCR4/ACKR3 interactomes and phosphorylation state on their function are discussed. Knowledge of the CXCR4/ACKR3 interactomes, along with their phosphorylation and ubiquitination status, sheds new light on their regulation and pathophysiological functions.

ACKR3 has emerged as a key player in several biologic processes and in particular during development. With its chemokine scavenging activity and atypical signaling properties, ACKR3 acts as a main regulator of physiologic processes at steady state and during inflammation. ACKR3 expression in mammalian tissues is outlined, and its role during development and potential contribution to the immune system of the myeloid lineage are discussed in another review (Koenen et al., 2019). Moreover, ACKR3 is overexpressed in numerous cancer types and has been involved in the modulation of tumor cell proliferation and migration, tumor angiogenesis, or resistance to drugs, thus contributing to cancer progression and metastasis. In that review, the clinical significance and potential mechanisms underlying the pathologic role of ACKR3 in breast, lung, and brain cancer are discussed (Neves et al., 2019). In addition, the possible relevance of ACKR3 as prognostic factor and potential therapeutic target is addressed in that context.

Altogether, these minireviews provide important insights and leads for therapeutic targeting of this class of oncogenic GPCRs. The early-stage researchers and principal investigators of the ONCORNET consortium brought their unique expertise to the network and participated in educational and research-based training. This has led to advances in the field of these oncogenic receptors and strengthened the international, intersectorial, and interdisciplinary community of GPCR drug discovery.

\section{Acknowledgments}

This minireview series is a joint activity by early-stage researchers and principal investigators from the European Union's Horizon2020 MSCA Program under Grant Agreement 641833 (Oncogenic G Protein-Coupled Receptor Network of Excellence and Training).

\section{References}

Adlere I, Caspar B, Arimont M, Dekkers S, Visser K, Stuijt J, de Graaf C, Stocks M, Kellam B, Briddon S, et al. (2019) Modulators of CXCR4 and CXCR7/ACKR3 function. Mol Pharmacol 96:737-752.

Arimont M, Hoffmann C, de Graaf C, and Leurs R (2019) Chemokine Receptor Crystal Structures: What Can Be Learned from Them? Mol Pharmacol 96: 765-777.

Bobkov V, Arimont M, Zarca A, De Groof T, van der Woning B, de Haard H, and Smit MJ (2019) Antibodies targeting chemokine receptors CXCR4 and ACKR3. Mol Pharmacol 96:753-764.

Fumagalli A, Zarca A, Neves M, Caspar B, Hill SJ, Mayor F, Smit MJ, and Marin P (2019) CXCR4/ACKR3 phosphorylation and recruitment of interacting proteins: key mechanisms regulating their functional status. Mol Pharmacol 96:794-808.

Heuninck J, Perpina Viciano C, Işbilir A, Caspar B, Capoferri D, Briddon SJ, Durroux T, Hill SJ, Lohse MJ, Milligan G, et al. (2019) Context-dependent signalling of CXC chemokine receptor 4 (CXCR4) and atypical chemokine receptor 3 (ACKR3). Mol Pharmacol 96:778-793.

Koenen J, Bachelerie F, Balabanian K, Schlecht-Louf G, and Gallego C (2019) Atypical chemokine receptor 3 (ACKR3): a comprehensive overview of its expression and potential roles in the immune system. Mol Pharmacol 96:809-818.

Neves M, Fumagalli A, van den Bor J, Marin P, Smit MJ, and Mayor F Jr (2019) The Role of ACKR3 in Breast, Lung and Brain Cancer. Mol Pharmacol 96:819-825.

O’Hayre M, Vázquez-Prado J, Kufareva I, Stawiski EW, Handel TM, Seshagiri S, and Gutkind JS (2013) The emerging mutational landscape of G proteins and G-protein-coupled receptors in cancer. Nat Rev Cancer 13:412-424.

Peled A, Wald O, and Burger J (2012) Development of novel CXCR4-based therapeutics. Expert Opin Investig Drugs 21:341-353.

Sánchez-Martín L, Sánchez-Mateos P, and Cabañas C (2013) CXCR7 impact on CXCL12 biology and disease. Trends Mol Med 19:12-22.

Address correspondence to: Dr. Martine J. Smit, VU University Amsterdam, De Boelelaan 1083, Amsterdam 1081 HV, Netherlands. E-mail: mj.smit@ vu.nl 